\title{
Thesen zur Ökonomie und Biologie des Vertrauens
}

Ernst Fehr

1. Ich betrachte Vertrauen als die Erbringung einer kritischen Vorleistung in der Erwartung/Hoffnung, dafür eine explizit vereinbarte oder eine implizite Gegenleistung zu erhalten. Die Gegenleistung kann, muss aber nicht, in Form ökonomischer Vorteile erbracht werden. Vertrauen ist daher im ökonomischen und sozialen Austausch - der ja fast immer eine zeitliche Distanz zwischen (Vor)leistung und Gegenleistung beinhaltet - allgegenwärtig. Wir unternehmen täglich »unzählige« Vertrauensakte.

2. Alle psychisch gesunden Menschen haben die Fähigkeit selbst fremden Menschen zu vertrauen. Diese Fähigkeit wird - wie unsere Forschung zeigt - durch Neurohormone, die unsere Hirnaktivitäten steuern, beeinflusst.

3. Die Vertrauenshandlung wird - auf individuell-psychologischer Ebene - durch die Risiko- und Betrugsaversion und durch die Einschätzung der Wahrscheinlichkeit, die gewünschte Gegenleistung zu erhalten, entscheidend beeinflusst.

4. Die individuelle psychologische Neigung zu Risikoaversion und Betrugsaversion wird durch biologische und gesellschaftliche Prozesse bestimmt. Man weiss aber noch sehr wenig über diese Einflüsse. Bei Erwachsenen Menschen ist davon auszugehen, dass diese Neigungen relativ stabil sind.

5. Wenn man daher das Vertrauen von Menschen oder gar ganzer Gruppen von Menschen beeinflussen will, dann muss das vor allem über die Beeinflussung der Erwartungen (d.h. der Wahrscheinlichkeitseinschätzung über die Gegenleistung) geschehen.

6. Vertrauen in andere Menschen und Institutionen erhöht man daher in erster Linie dadurch, dass man diese vertrauenswürdiger macht. Es gibt im Wesentlichen drei Quellen der Vertrauenswürdigkeit von Menschen: 1) Die Existenz von expliziten Vorschriften, Gesetzen und Regeln, deren Einhaltung mit positiven oder negativen Sanktionen gesichert wird. 2) Die Existenz von informellen Regeln und Reputationsanreizen für vertrauenswürdiges Verhalten. 3) Die individuelle Moral von Menschen. 
7. Gesetze und Vorschriften: Die Tatsache, dass der Staat viele Verträge durch geeignete Institutionen (Polizei, Gerichte, etc.) durchsetzt, erhöht die Bereitschaft zum Tausch, also zur Erbringung kritischer Vorleistungen.

8. Reputationsanreize: Die Tatsache, dass das Betrügen des Tauschpartners dem guten Ruf schadet und daher die zukünftigen Tausch- und Gewinnmöglichkeiten einschränkt, erhöht die Anreize zur Einhaltung von Tauschbedingungen, und damit auch die Bereitschaft zu vertrauen.

9. Individuelle Moral: Die Tatsache, dass eine Person abgegebene Versprechen einhält, selbst wenn sie einen Nettovorteil aus dem Bruch des Versprechens ziehen würde, macht diese Person zu einem vertrauenswürdigen Partner, und erhöht das Vertrauen anderer Menschen in sie.

10. Der Vertrauensakt hat häufig selbst eine nutzenerhöhende Wirkung, da die Menschen es schätzen, wenn Ihnen vertraut wird. Vertrauen ist aber nicht von Dauer, wenn es nicht von der Vertrauenswürdigkeit des Partners/der Institution gestützt wird. Deshalb hat das gesellschaftliche Vertrauen in Menschen und Institutionen nur in eingeschränktem Masse eine positive Auswirkung auf die gesamtgesellschaftliche Wohlfahrt. Entscheidend ist vielmehr die Vertrauenswürdigkeit von Menschen und Institutionen.

- Prof. Dr. Ernst Fehr leitet das Department of Economics der Universität Zürich und war Co-Leiter des interdisziplinären Projektes »Vertrauen verstehen« und Leiter der Teilprojekte ,Die neurobiologischen, kognitiven und emotionalen Ursachen von Vertrauen. 